\title{
Lifetime Extension of RF MEMS Direct Contact Switches in Hot Switching Operations by Ball Grid Array Dimple Design
}

\author{
Linda L. W. Chow, Student Member, IEEE, John L. Volakis, Fellow, IEEE, Kazuhiro Saitou, Member, IEEE, \\ and Katsuo Kurabayashi, Member, IEEE
}

\begin{abstract}
Direct contact RF microelectromechanical systems switches have demonstrated excellent ultrawideband performance from dc to $100 \mathrm{GHz}$. However, they are prone to failures due to contact adhesion and arcing, particularly for pure-gold/pure-gold contacts. In this letter, we present a new contact design employing ball grid array (BGA) dimples that limit the effective contact area to a few tens of nanometers in diameter. We experimentally show the performance of the BGA dimple with pure-gold/pure-gold contacts and demonstrate RF power handling greater than $1 \mathrm{~W}$ during hot switching in excess of $\mathbf{1 0 0}$ million cycles.
\end{abstract}

Index Terms-Life estimation, material reliability, microelectromechanical devices, microwave switches, power transmission reliability.

\section{INTRODUCTION}

$\mathbf{R}$ ECONFIGURABLE RF systems that are intended to operate in multiple-frequency bands require reliable switching devices. Direct metal contact RF microelectromechanical systems (MEMS) switches are promising candidates due to their wide bandwidth, high isolation, low insertion loss, and linearity [1]. However, these advantages are often overshadowed by limited RF power-handling capability and short lifetime. Primary factors for their failure are metal-metal adhesion and contact surface degradation, resulting in unacceptably high contact resistance $(>1 \mathrm{k} \Omega)$ [2], [3]. To improve the longterm reliability, we hereby propose an innovative contact design employing a grid array of ball-shaped dimples across the metal-metal interface.

Our contact dimple design concept was initiated based on our previous findings: 1) A smaller apparent contact area yields less contact adhesion [4]; 2) contact resistance can be as small as $0.1 \Omega$ with a submicrometer effective contact area of $\sim 0.1 \mu \mathrm{m}^{2}$ [5]. With its constricting tip, a ball-shaped dimple has a much smaller apparent contact area, leading to less adhe-

Manuscript received February 21, 2007; revised March 15, 2007. This work was supported by the National Science Foundation under Grant ECS 0330963. The review of this letter was arranged by Editor J. Sin.

L. L. W. Chow, K. Saitou, and K. Kurabayashi are with the Department of Mechanical Engineering, University of Michigan, Ann Arbor, MI 48109-2125 USA (e-mail: lindchow@umich.edu; kazu@umich.edu; katsuo@umich.edu).

J. L. Volakis is with the Department of Electrical and Computer Engineering, Ohio State University, Columbus, OH 43212 USA (e-mail: volakis@ ece.osu.edu).

Color versions of one or more of the figures in this letter are available online at http://ieeexplore.ieee.org.

Digital Object Identifier 10.1109/LED.2007.896811 sion than a flat dimple of identical diameter (typically found in conventional MEMS switches) while maintaining sufficiently small contact resistance; and 3) a ball-grid-array (BGA) contact alters the switch conduction locations from dimples to other dimples in hot switching, thus reducing the probability of local material degradation [6].

This letter aims to improve switch reliability in hot-switching operations, where switch reliability is significantly deteriorated since material evaporation and arcing are promoted at heated asperities like field emitters [7]. If the contact dimple has a ball shape, high electric fields that could cause arcing only occur near its tip, whereas the sharp edges and larger apparent contact area of a flat dimple may induce greater arcing. We propose a grid dimple design also because closely packed surface extrusion arrays are known to decrease field emissions due to screening effects [8]. Specifically, we hypothesize that having a high packing dimple density (2- $\mu \mathrm{m}$ pitch), the BGA design could suppress the arcing-induced contact failure with field screening as well as the local mechanical deformation of each dimple. To test our hypothesis and show the improved contact reliability, we compare the lifetime of BGA switch contacts to those of other dimple designs.

\section{EXPERIMENTS}

Sample switches of pure sputtered gold (99.99\% purity) were fabricated by employing our low-temperature $\left(<160^{\circ} \mathrm{C}\right)$ process [6]. Here, we mainly considered two dimple designs: 1) ball shaped with a footprint of $1.5 \mu \mathrm{m}^{2}$ and 2) flat surfaced with a footprint of $3.7 \mu \mathrm{m}^{2}$. Both designs were tested in single and array/grid configurations. Fig. 1 shows scanning electron microscopy (SEM) images of the ball-shaped dimples in single (S1) and grid (G1) configurations. The flat dimples are also shown in single (S2) and grid (G2) configurations. Having a diameter of $1 \mu \mathrm{m}$, both $\mathrm{S} 1$ and G1 yield submicrometer contacts.

A minimum switch actuation voltage of $60 \mathrm{~V}$ was found so as to suppress surface degradation due to cyclic mechanical impacts. It was determined by slowly increasing the supplied voltage from zero until the contact first became conductive, and it was used during all subsequent hot-switching experiments. Employing a static pure bending analysis of an Euler-Bernoulli beam, the contact and restoring forces were estimated to be $<20$ and $\sim 40 \mu \mathrm{N}$, respectively. The dynamic bouncing of the 


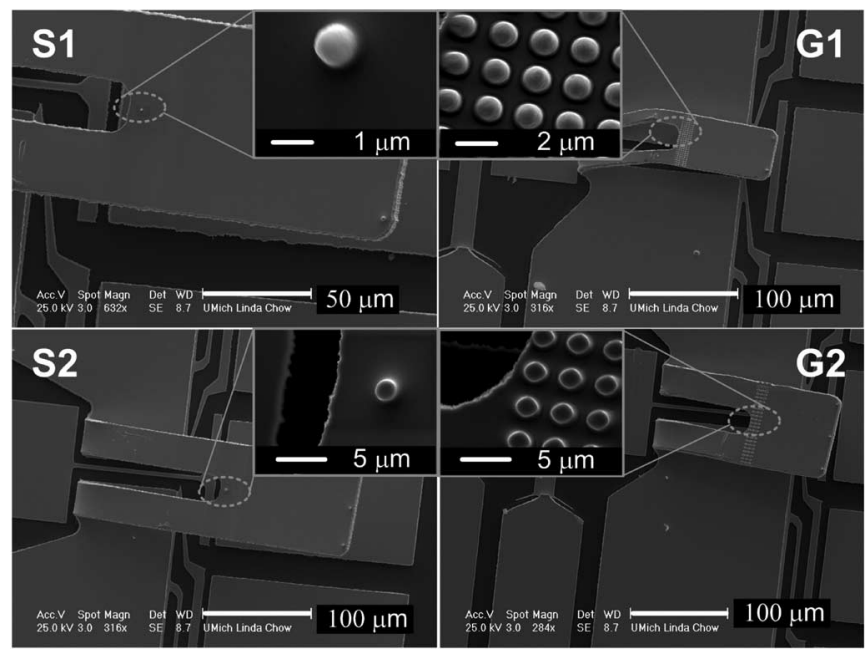

Fig. 1. SEM images of ball-shaped dimples (G1 and S1) and flat-surfaced dimples (G2 and S2) after flipping the cantilever switches upside down. G1 and $\mathrm{G} 2$ have 300 and 60 dimples, respectively. The images were taken before the $\mathrm{RF}$ testing.

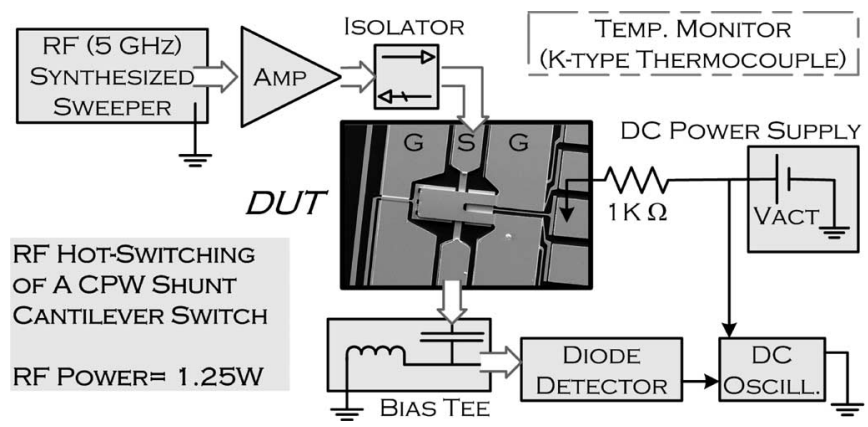

Fig. 2. Schematic setup for measuring the switching time of shunt MEMS switches in RF hot switching.

beam might yield a contact force that is five times as large as the static value [9]. It has been shown that a hydrocarbon insulating film exists on the contact surface [10], causing unacceptably large contact resistance in typical low-contact-force $(<100 \mu \mathrm{N})$ operation for RF MEMS switches. To mitigate this, we applied a softening voltage of $60 \mathrm{mV}$ at a dc four-wire $I-V$ setup in the beginning of the switch operation, and this reduced the contact resistance to $\sim 0.5 \Omega$. This softening voltage, which depends on the contact material and initial contact resistance, was carefully selected not to cause any significant adhesion following a process used in previous experiments [11].

The hot-switching performance was measured at an RF power input of $1.25 \mathrm{~W}$ using the experimental setup that is shown in Fig. 2. The RF power at $5 \mathrm{GHz}$ (synthesized by an HP 83624A) was amplified and fed through the switches, whereas the voltage across the contact was displayed on a dc oscilloscope (HP 54645A) using a diode detector (Krystar zero-bias Schottky detector 202B) to carry out the RF-dc conversion. The switches were actuated separately using a dc power supply (HP $3245 \mathrm{~A})$ at a switching frequency of $5 \mathrm{kHz}$. The oscilloscope was triggered to rising slope traces such that the switching response appeared as standing waves. Monitoring of the timedomain traces of the contact voltage using the oscilloscope allowed us to ensure that the switch was in the "ON" and "OFF"

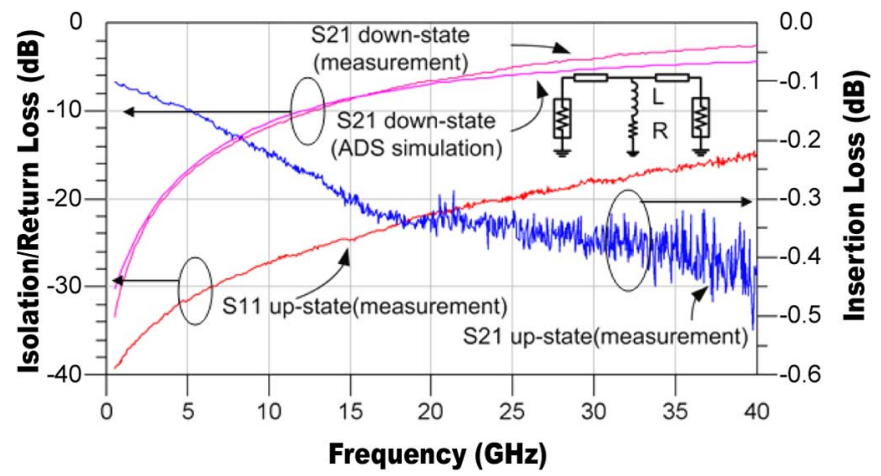

Fig. 3. Typical RF characteristics of testing direct contact shunt switches showing isolation (down-state $S_{21}$ ), insertion (up-state $S_{21}$ ), and return $\left(S_{11}\right)$ losses.

states at each actuation cycle while measuring the switching time over $10^{5}$ cycles. Our observation of consistent on/off switching without significant changes confirmed that there was no delay due to possible contact stiction or self-release during RF testing.

\section{RESUlTS AND DiscusSiONS}

Fig. 3 shows typical $S$-parameter plots that are measured with the sample switches from 0.5 to $40 \mathrm{GHz}$ for the up state (insertion-loss state) and the down state (isolation-loss state). As the dimple area occupies only $1 / 1000$ of the signal wavelength, different dimple designs do not show noticeable variation on the $S$-parameter plots. It can be seen that these dimples work well with small insertion and return losses from dc to $15 \mathrm{GHz}$.

When no contact failure occurred, all switches showed similar switching behaviors in terms of their opening $(<2 \mu \mathrm{s})$ and closing $(<50 \mu \mathrm{s})$ responses. The slower closing behavior is attributed to several bouncing motions of the switch beam before it settles on the down state. Hot-switching cycles were counted by recording the time to failure. After hot-switching measurements (using over 20 samples for each design), we observed that only the BGA dimples (G1) were still working after 100 million cycles. The switches with the single ball-shaped dimple eventually experienced contact adhesion possibly due to mechanical deformation of the dimple. The flat dimple designs (S2 and G2) remained open (failed) after about one million switching cycles, as summarized in Fig. 4(a).

To obtain some insight into the root causes, we flipped the switch cantilevers and studied the surface morphology of their contact dimples using SEM. Wire-like protrusions were found near the edges of some flat dimples, as in Fig. 4(b). Energy dispersive X-ray analysis confirmed that these protrusions were pure gold structures about $100 \mathrm{~nm}$ in diameter, cylindrical in shape, and thicker at their roots. It is likely that these protrusions were caused by local melting and elongation of gold during the high-power cycling. However, no such protrusions were observed on the BGA switches (G1). Furthermore, explosive material removal (under the contact) was also observed in some flat dimples, as shown in Fig. 4(c). As such, we believe that the nanoscale protrusion in Fig. 4(b) can be the precursor 


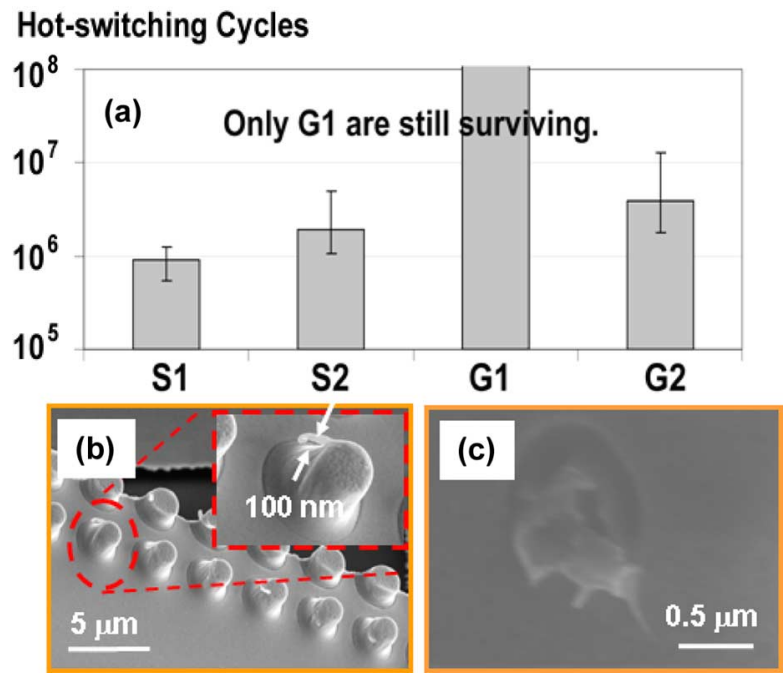

Fig. 4. (a) Average lifetimes for different dimple design groups. (b) Wire-like protrusions from flat-surfaced dimple arrays. (c) Explosive material removal at a single flat-surfaced dimple. The error bars in (a) represent the standard deviation among 20 sample data that were obtained for each dimple design.

for vaporization-induced arcing in high electric fields when the contacts are positioned very close to the substrate surface. This leads to the subsequent permanently open failures. Nonetheless, the BGA dimple design (G1) never suffered from the abrupt contact damage that is caused by the explosive material removal, supporting our working hypothesis in Section I. Further research is underway to obtain a better understanding of the physics leading to the improvements brought forward by the BGA structure.

\section{CONCLUSION}

The proposed BGA dimple design allows for fast switching speed (in microseconds), good power handling $(>1 \mathrm{~W})$, and extended lifetime ( $>100$ million hot-switching cycles) with low-temperature fabrication $\left(<160{ }^{\circ} \mathrm{C}\right)$. The proposed dimple design holds the promise to highly improve the performance and reliability of RF MEMS contacts by simply changing the mask layout without changing the contact materials and device processes. A good understanding of the arcing failure-causing mechanism awaits further investigations.

\section{REFERENCES}

[1] G. M. Rebeiz, RF MEMS Theory, Design and Technology, 1st ed. Hoboken, NJ: Wiley, 2003

[2] P. M. Zavracky, S. Majumder, and N. E. McGruer, "Micromechanical switches fabricated using nickel surface micromaching," J. Microelectromech. Syst., vol. 6, no. 1, pp. 3-9, Mar. 1997.

[3] S. Majumdar, N. E. McGruer, G. G. Adams, P. M. Zavracky, R. H. Morrison, and J. Krim, "Study of contacts in an electrostatically actuated microswitch," Sens. Actuators A, Phys., vol. 93, no. 1, pp. 19-26, Aug. 2001.

[4] B. D. Jensen, K. Huang, L. L. W. Chow, and K. Kurabayashi, "Adhesion effects on contact opening dynamics in micromachined switches," J. Appl. Phys., vol. 97, no. 10, p. 103 535, May 2005.

[5] B. D. Jensen, K. Huang, L. L. W. Chow, and K. Kurabayashi, "Lowforce contact heating and softening using micromechanical switches in diffusive-ballistic electron transport transition," Appl. Phys. Lett., vol. 86 , no. 2, p. 023 507, Jan. 2005.

[6] L. L. W. Chow, S. A. Schrader, and K. Kurabayashi, "Transition from multiple to single microcontact conduction during hot switching of microelectromechanical switches with ball-shaped dimples," Appl. Phys. Lett., vol. 89, no. 13, p. 133 501, Sep. 2006.

[7] F. Charbonnier, "Arcing and voltage breakdown in vacuum microelectronics microwave devices using field emitter arrays: Causes, possible solutions, and recent progress," J. Vac. Sci. Technol. B, Microelectron. Process Phenom., vol. 16, no. 2, pp. 880-887, Mar. 1998.

[8] A. Korotocov, Y. S. Huang, T. Y. Tsai, D. S. Tsai, and K. K. Tiong, "Effect of length, spacing and morphology of vertically aligned $\mathrm{RuO}_{2}$ nanostructures on field-emission properties," Nanotechnology, vol. 17, no. 13, pp. 3149-3153, Jul. 2006.

[9] B. McCarthy, G. G. Adams, N. E. McGruer, and D. Potter, "A dynamic model, including contact bounce, of an electrostatically actuated microswitch," J. Microelectromech. Syst., vol. 11, no. 3, pp. 276-283, Jun. 2002.

[10] D. Hyman and M. Mehregany, "Contact physics of gold microcontacts for MEMS switches," IEEE Trans. Compon. Packag. Technol., vol. 22, no. 3, pp. 357-364, Sep. 1999.

[11] B. D. Jensen, L. L. W. Chow, K. Huang, K. Saitou, J. L. Volakis, and K. Kurabayashi, "Effect of nanoscale heating on electrical transport in RF MEMS switch contacts," J. Microelectromech. Syst., vol. 14, no. 5, pp. 935-946, Oct. 2005. 